\title{
Lens Pit
}

National Cancer Institute

\section{Source}

National Cancer Institute. Lens Pit. NCI Thesaurus. Code C34201.

A depression in the surface ectoderm in the head region of the early embryo formed by invagination of the lens placodes, which houses the lens precursor cells. 